\title{
A Comprehensive Study on Cloud Computing and their Service Models
}

\author{
Bobbadi Manasa \\ Assistant Professor,
}

Department of IT, University College of Engineering, JNTUK, Vizianagaram.

\section{Abstract:}

In this paper it mainly discuss some important issues about both private and public clouds, mainly these public and private cloud are maintained by the IT departments, and it mainly describes the cloud computing platforms. Private cloud offers same features that present in public clouds but private clouds provides more secure process. If we maintain the private clouds it provides more security than public clouds because private clouds are handled by only the particular enterprise which build it so, that there is no sharing mechanism so, there will be fewer burdens than other type of clouds.

Keywords-- cloud computing, private clouds, public clouds.

\section{INTRODUCTION:}

\section{Definition:}

Cloud computing is one of the technique that can be used by the hardware and software to create a service or platform at anywhere, any time and any place over the internet.

Cloud computing is ability to deliver a on demand computing services over the internet. It is a computing resources which is used to store the database, applications services, server information and on demand network access by the service provider provided by the internet[1][2]..

To access the internet users can apply many applications and advanced operating environments by using the cloud computing. Cloud computing is used to refer the internet based developments services.

Cloud computing is a service that provides or optimizes mainly based on data center to provide software, hardware and information resources. Organizations can simply connected a cloud and use the resources which are available there[1][2].

We can collect or use resources by low cost using cloud by taking 2 different models. They are,

1) Deployment cloud

2) Service cloud

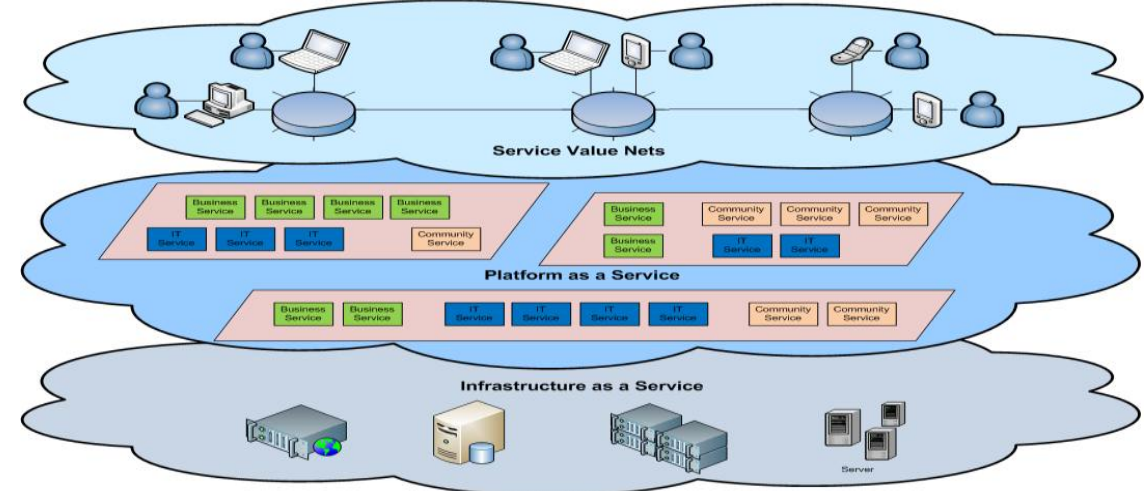

Fig.1: Block Diagram 


\section{Deployment model:}

In deployment model there are 3 types of clouds. They are
1) Public cloud
2) Private cloud
3) Hybrid cloud

Public cloud: public cloud is a computing service which is available on the public internet[3][4][5].

\section{Advantages:}

- Reduces cost

- Highly scalable

- Provides universal accessibility

- Applications and data are automatically upgraded.

\section{Example:}

BUS: which is accessible to anyone with less cost.

Private cloud:

Private clouds can be used and developed for only one organization and it is restricted to some people who get membered in that organization.

Example:

OWN CAR: if we buy a car then we will maintained by owner car.

There are two kinds of private clouds.

1). On premises internet cloud

2). externally hosted virtual private cloud[3][4][5].

On premises internet cloud: In internet private clouds, any company or organization can access the internet through data and systems are placed in private cloud only.

Externally hosted cloud: any company or organization can access the data and systems in a hosted virtual private cloud through internet. These data is interlinked with the cloud service provider[3][4][5].

Hybrid Cloud: It is the combination of characteristics and flexibility of both public and private clouds.

Here any company can access data which os presenting on the onprimises rivate cloud directly and also access some data in hosted virtual private cloud which is interlink with cloud service providers through the internet[3][4][5].

Example: We can take a private taxi by rental, if we pay high amount then we can make a journey comfortable otherwise we can share with another person if we want to pay less amount.

\section{Characteristics of cloud: \\ Ondemand service:}

We can use data at anywhere, at any time in any platform. We can use the system or we can retrieve the data from cloud when you needed to perform a task[3][4][5].

\section{Network access:}

To use the data which is presenting on the cloud we should use internet as a medium to retrieve or access any information. 
Shared resourses: Resources are combined together and they can used by multiple clients or multiple users.

Scalability: Scalability allows elasticity of the available resources.

DIFFERENCES BETWEEN CLOUD COMPUTING AND ONPRIMISES SYSTEM NETWORK.

\section{ADVANTAGES OF CLOUD COMPUTING:}

- Pay for what you use

Scale up=pay more

Scale less=pay less.

- No server space required

- No experts required for hardware and software maintenance

- Better data security

- Disaster recovery

- High flexibility

Easily undo, migrate to new environment

- Automatic software updates

- Teams cn collaborate from widespread locations

- Data can be shared and accessed anywhere over the internet

- Rapid implementation

\section{On primises system network:}

Examples of companies that can use cloud computing:

1. Aws (Amazon Web Server)

2. I Cloud By Apple Products

3. Microsoft Azure By Microsoft

4. Google Cloud

5. Ibm Smart Cloud.

\section{Cloud Service Models:}

There are 3 types of cloud service models..

\section{They are}

1. Software as a service

2. Platform as a service

3. Infrastructure as a service[3][4][5]

\section{SOFTWARE AS A SERVICE:}

It is software that offers on demand pre produced pay per use of application software to the users. This service is platform independent because we don't need to install the software on our PC of system. the cloud runs a single instance of a software tht is available for multiple end users. All the computing resources managed by vendor this makes cloud computing became cheap. This typr of services can be accessible via a web browser or light weight client applications [3][4][5].

Finally, software as a service produces or delivers computer infrastructure, build data centers, managing power, scale, hardware, networking, storage, distributed systems etc 
ratherthan purchasing servers, datacenter space or networking equipment, clients instead of buying those resources, as a fully outsourced services[3][4][5].

Example: Amazon web service, VM ware, azure, rack space hosting.

Software as a service is a licensing on subscription basis and is centrally hosted sometimes it will referred as ON DEMAND SOFTWARE. Software as a service typically accessed by the user via a web browser. SAAS is become a common delivery model for many business applications including,

- Office managing software

- Data base management system software

- Development software

- Collaboration

- Content management

- Service desk management

It has been incorporated into strategy of all leading enterprise software companies.

\section{PROS:}

- Universal accessible for any platform

- No need to commute, you can work from any place.

- Excellent for collaborative working.

- Vendor provides modest software tools.

- Allows for multi-tenancy.

\section{CONS:}

- Portability and browser issues

- Internet performance may dictate overall performance

- Compliance restrictions.

\section{PLATFORM AS A SERVICE:}

This service is nade up of a programming language execution environment like an operating system, a web browser and a database. All of these can encapsulate the environment where users can built, compile and run their programs without worrying of the underlying infrastructure. In this model, you can manage data and the application recourses and all other recourses are managed by vendor[6][7][8].

PAAS provides developers necessary tools to create, test any application, host and maintain those created applications. Cloud providers offers an internet based platform to developers who can create services but donot want to built their own cloud.

Example: Microsoft Azure, googlle apps, AWS.

Platform as a service is a category of cloud computing service that provides a platform and allowing customers to develop, run and manage applications. Customers can be always with an complexity of building and maintaining the infrastructure. Typically PAAS associated with developing and launching an application[6][7][8].

PAAS can be delivered in 2 ways.

\section{AS A PUBLIC CLOUD SERVICE FROM A PROVIDER :}

In this customer controls software deployment and configuration settings whereas provider provides networks, servers, storage and other service to host the customers applications[6][7][8]. 


\section{AS A SOFTWARE INSTALLED IN PRIVATE DATA CENTERS :}

This is managed by internal IT departments.PAAS can be simplifies the code writing process for developers, with the infrastructure and operations handled by the providers. Originally all the platforms were in the public cloud because many companies developed want to have everything in their private cloud and hybrid options were created[6][7][8].

PAAS provides an environment for developers and companies to create, host and deploy applications from the complexities of the infrastructure.

Example: setting up configuration.

Advantages:

- PAAS can improves the speed of developing an application.

- Allows the consumer to focus on the application itself.

- With PAAS consumer can manages an application and data while the provider manages runtime, middleware, operating system, virtualization, servers, storage and networking.

- The development tools which are provided by the vendor can manages the customized according to the needs of the user.

- The user can choose to maintain the software or have the vendor maintain it.

- PAAS may also include some facilities for application design, application development and deployment.

PAAS related services include:

- Web server integration

- Data base integration

- Security

- Scalability

- Storage

- Application instrumentation.

- Includes mechanisms for service management such as monitoring, workflow, discovery and reservation[6][7][8].

\section{PROS:}

1. Cost effective rapid development

2. Faster market for developers.

3. Easy development of web applications

4. Private or public deployment is possible.

\section{CONS:}

1. Developers are limited to the providers languages and tools.

2. Migration issues - such as the risk over the vendor lock-in.

\section{Infrastructure As A Service:}

This service offers the computing architecture and infrastructure. There is all computing resourses present in a virtual environment so that multiple users can access them[9][10]. Resourses include :

- Data storage

- Virtualization

- Servers

- Networking. 
DOI: https://dx.doi.org/10.26808/rs.ca.i8v5.04 International Journal of Computer Application (2250-1797)

Issue 8 Volume 5, Sep. - Oct. 2018

Most of the vendors are responsible for managing these four resources. User will responsible for managing other resources such as applications, data, runtime and middleware. Mainly system admins are the users of IAAS[9][10].

\section{PROS :}

- The cloud provides the infrastructure.

- Enhanced scalability

- Dynamic workloads are supported

- IAAS is flexible.

\section{CONS:}

- Security issues

- Network and service delays.

\section{CONCLUSION:}

The above sections describes what are public cloud and private clouds and how far they provide security for thedata and we have also seen the storage capabilities of public and private clouds and each of them have their ownsignificance and we have also seen the key features of the public and private storages. And we have also came to know how to build private and public clouds and what steps should follow while building them. If we are using the private cloud then we should have own infrastructure and low bandwidth and expensive. If we want change it to the public clouds we have refines all the web services and more infrastructures are needed and high bandwidth, cheaper. Based on the requirement we have select right choice, if there is a requirement of both public and private clouds, it is better to opt hybrid cloud. A Hybrid Cloud is combination of public cloud storage and private cloud storage where some critical data resides in the enterprise's private cloud while other data is stored and accessible from a public cloud storage provider. For combining both public and private clouds both resources should be manages. So that Hybrid cloud works efficiently

\section{References:}

[1] https://www.youtube.com/watch?v=p7wSwb4Gv20

[2] https://www.youtube.com/watch?v=36zducUX16w

[3] https://www.youtube.com/watch?v=Zib9aP1TsZ8

[4] https://www.youtube.com/watch?v=SgujaIzkwrE

[5] https://www.paranet.com/blog/bid/128267/the-three-types-of-cloud-computingservice-models

[6] https://www.youtube.com/watch?v=whx01vzmlns

[7] https://www.youtube.com/results?search_query=platform+as+a+service+explained

[8] https://www.youtube.com/watch?v=whx01vzmlns

[9] https://www.youtube.com/results? search_query=inffrastructure+as+a+service

[10] https://www.youtube.com/results?search_query=infrastructure+as+a+service+explai ned

[11] https://www.youtube.com/results?search_query=software+as+a+service+as+a+servic $\mathrm{e}$

[12] https://www.youtube.com/results?search_query=software+as+a+service+explained

[13] https://azure.microsoft.com/en-in/overview/examples-of-cloud-computing/

[14] https://www.technobuffalo.com/2010/01/17/five-examples-of-cloud-computing/

[15] Eswar et al./ IJEA “A Study on Building Private and Public Clouds and their storage details in cloud computing" Vol.1 Issue 6 ISSN:2320-0804 